\title{
QuickBrain MRI for the detection of acute pediatric traumatic brain injury
}

\author{
David C. Sheridan, MD, MCR, ${ }^{1}$ Craig D. Newgard, MD, MPH, ${ }^{1}$ Nathan R. Selden, MD, PhD, ${ }^{2}$ \\ Mubeen A. Jafri, MD, ${ }^{3}$ and Matthew L. Hansen, MD, MCR ${ }^{1}$
}

${ }^{1}$ Department of Emergency Medicine, Center for Policy and Research in Emergency Medicine, ${ }^{2}$ Department of Neurological Surgery, Division of Pediatric Neurosurgery, and ${ }^{3}$ Department of Surgery, Division of Pediatric Surgery, Oregon Health \& Science University, Portland, Oregon

\begin{abstract}
OBJECTIVE The current gold-standard imaging modality for pediatric traumatic brain injury (TBI) is CT, but it confers risks associated with ionizing radiation. QuickBrain MRI (qbMRI) is a rapid brain MRI protocol that has been studied in the setting of hydrocephalus, but its ability to detect traumatic injuries is unknown.

METHODS The authors performed a retrospective cohort study of pediatric patients with TBI who were undergoing evaluation at a single Level I trauma center between February 2010 and December 2013. Patients who underwent CT imaging of the head and qbMRI during their acute hospitalization were included. Images were reviewed independently by 2 neuroradiology fellows blinded to patient identifiers. Image review consisted of identifying traumatic mass lesions and their intracranial compartment and the presence or absence of midline shift. CT imaging was used as the reference against which qbMRI was measured.
\end{abstract}

RESULTS A total of 54 patients met the inclusion criteria; the median patient age was 3.24 years, $65 \%$ were male, and $74 \%$ were noted to have a Glasgow Coma Scale score of 14 or greater. The sensitivity and specificity of qbMRI to detect any lesion were $85 \%$ (95\% Cl 73\%-93\%) and 100\% (95\% Cl 61\%-100\%), respectively; the sensitivity increased to $100 \%$ $(95 \% \mathrm{Cl} 89 \%-100 \%)$ for clinically important TBIs as previously defined. The mean interval between CT and qbMRI was 27.5 hours, and approximately half of the images were obtained within 12 hours.

CONCLUSIONS In this retrospective pilot study, qbMRI demonstrated reasonable sensitivity and specificity for detecting a lesion or injury seen with neuroimaging (radiographic TBI) and clinically important acute pediatric TBI.

https://thejns.org/doi/abs/10.3171/2016.7.PEDS16204

KEY WORDS trauma; imaging; child

$\mathrm{P}$ EDIATRIC head trauma remains a frequent cause of presentation to the emergency department (ED). ${ }^{12} \mathrm{~A}$ majority of children with head injury present to the ED with mild symptoms and a Glasgow Coma Scale (GCS) score of 14 or 15 , which indicates a relatively low risk of clinically important traumatic brain injury (ciTBI) $.^{10} \mathrm{How}-$ ever, because of the potential consequences of missed intracranial injury, CT imaging is frequently used to further evaluate such patients. Recent studies have found dramatic increases in the rate of CT imaging. ${ }^{1}$ A recent Pediatric Emergency Care Applied Research Network (PECARN) study addressed this issue and developed a highly sensitive clinical decision rule that identified children at low risk of ciTBI, which is different from a lesion or injury seen on neuroimaging (radiographic TBI) and might not need necessitate head imaging. ${ }^{12}$ In many clinical scenarios, this rule has the potential to reduce CT use substantially. However, even when this rule is applied, the results of a majority of CT scans are negative, which indicates that children are being exposed unnecessarily to ionizing radiation.

Magnetic resonance imaging does not use ionizing radiation and has been shown to be accurate for detecting

ABBREVIATIONS ciTBI = clinically important traumatic brain injury; ED = emergency department; GCS = Glasgow Coma Scale; GRE = gradient echo; $\mathrm{LOS}=$ length of stay; PICU = pediatric intensive care unit; qbMRI = QuickBrain MRI; TBI = traumatic brain injury.

SUBMITTED April 12, 2016. ACCEPTED July 28, 2016.

INCLUDE WHEN CITING Published online November 25, 2016; DOI: 10.3171/2016.7.PEDS16204. 
TBI. ${ }^{13,19}$ However, MRI takes longer to perform than CT, requires patients to hold still for an extended time, and often requires sedation, all of which limits its use in pediatric patients. QuickBrain MRI (qbMRI) includes rapid acquisition of axial, sagittal, and coronal T2-weighted fast spin echo images. Images can be obtained in approximately 1-3 minutes. qbMRI was studied previously to evaluate ventriculoperitoneal shunt failure in children with hydrocephalus and was found to equal the accuracy of CT; only approximately $4 \%$ of patients in both the CT and MRI groups needed an anxiolytic/sedative. ${ }^{21}$ According to a recent survey, approximately $80 \%$ of institutions with a pediatric neurosurgeon on staff have qbMRI capabilities, but the modality's clinical utility remains uncertain because more than $97 \%$ of these institutions still use CT for the evaluation of pediatric head trauma. ${ }^{11,15,20}$ However, the effectiveness of qbMRI in diagnosing ciTBI in the acute setting is unknown. The primary objective of this pilot study was to assess the accuracy of qbMRI in detecting acute radiographic TBI in pediatric patients.

\section{Methods}

This was a retrospective cohort study of pediatric patients who presented to the ED for an evaluation of head trauma. The Oregon Health \& Science University Institutional Review Board reviewed and approved this study and waived the requirement for informed consent. We conducted the study at a single Level I trauma center affiliated with a children's hospital in Portland, Oregon, from February 2010 to December 2013. This institution is 1 of 2 Level I trauma centers and 1 of 2 children's hospitals that serve the state of Oregon. The 2 trauma centers care for children and adults entered into the state trauma system according to their geographic designation in the Portland metropolitan area. The majority of emergency medical services transports directly from the scene of injury come from urban settings, and trauma transfers are often from rural areas of the state.

We screened all pediatric patients with trauma who were younger than 15 years (the age-based definition of a child in the Oregon Trauma System) and were cared for in the formal trauma system at our institution between February 2010 and December 2013. February 2010 was the time at which qbMRI began being used routinely for follow-up imaging in cases of TBI in our pediatric intensive care unit (PICU). We included patients who also underwent qbMRI during their acute hospital stay. We excluded patients who required an open neurosurgical procedure before qbMRI or patients for whom we could not access the initial head CT images from an outside hospital.

The principal investigator (D.C.S.) abstracted all clinical data from the electronic medical record (EPIC 2010), including sex, age at arrival, whether the patient was transferred or presented to the study institution for initial evaluation, GCS score on arrival, PICU length of stay (LOS), total hospital LOS, whether the patient was intubated endotracheally (including duration in days), whether a neurosurgical procedure was performed, death while in hospital, and the time interval (in hours) between head CT and qbMRI. ciTBI was defined as previously described to include intubation duration greater than 24 hours, total hospital LOS greater than 48 hours, need for a neurosurgical procedure, and/or death. ${ }^{1}$

QuickBrain MRI has been used routinely at our institution to perform follow-up imaging in all pediatric patients with TBI during their hospitalization. In addition, a number of pediatric patients with spinal cord conditions can undergo qbMRI for evaluation but in fact have no intracranial lesion; therefore, they represent a population with negative head-imaging results. CT and qbMRI were reviewed for the presence of an intracranial lesion, characterization of the location of the lesion (if present) as intraaxial or extraaxial, and whether midline shift was seen. All images were deidentified and placed in discreet folders within our imaging system before review. The head $\mathrm{CT}$ and qbMR images were then reviewed independently by 2 neuroradiology fellows ( 1 board certified in radiology and 1 board eligible in radiology) who could not link the images to individual patients. If a discrepancy between the 2 reviews was present, the formal attending neuroradiology interpretation of the study as documented in the medical record was reviewed and used in conjunction with the attending pediatric neurosurgical consultation note to resolve disagreements in interpretation by the study radiologists. All lesions missed on qbMRI were reviewed by an unblinded study pediatric neurosurgeon. Clinical and image review data were entered directly into an electronic database by the principal investigator (clinical data) or neuroradiology fellows (image review) independently.

\section{Outcomes}

The primary outcomes were the sensitivity and specificity of qbMRI in detecting pediatric TBI that had already been confirmed by CT. The secondary outcomes included the sensitivity of qbMRI to detect ciTBI, the sensitivity and specificity of qbMRI to detect midline shift, and characterization of the intracranial compartment containing the mass lesion as intraaxial or extraaxial. For all measures, head CT was used as the reference against which qbMRI was measured.

\section{Statistical Analysis}

We used descriptive statistics to characterize the sample and use of imaging modalities. We calculated the sensitivity and specificity of qbMRI for pediatric TBI by first comparing the results to CT-based radiographic findings and then by evaluating them for clinically important outcomes (secondary outcome). We used the Wilson-score method for binomial proportions to calculate $95 \%$ CIs for sensitivity and specificity.

\section{Results}

\section{Characteristics of Study Subjects}

A total of 54 patients met full inclusion criteria (Fig. 1). The median age of the cohort was 3.24 years (range $0.03-12.3$ years), and $65 \%$ were male. The majority $(82 \%)$ of the patients were transferred from another hospital. The GCS score was 14 or greater in 40 (74\%) patients; 9 (17\%) patients had a GCS score less than 8. ciTBI was present in 34 (63\%) patients, and no children died during their hospi- 


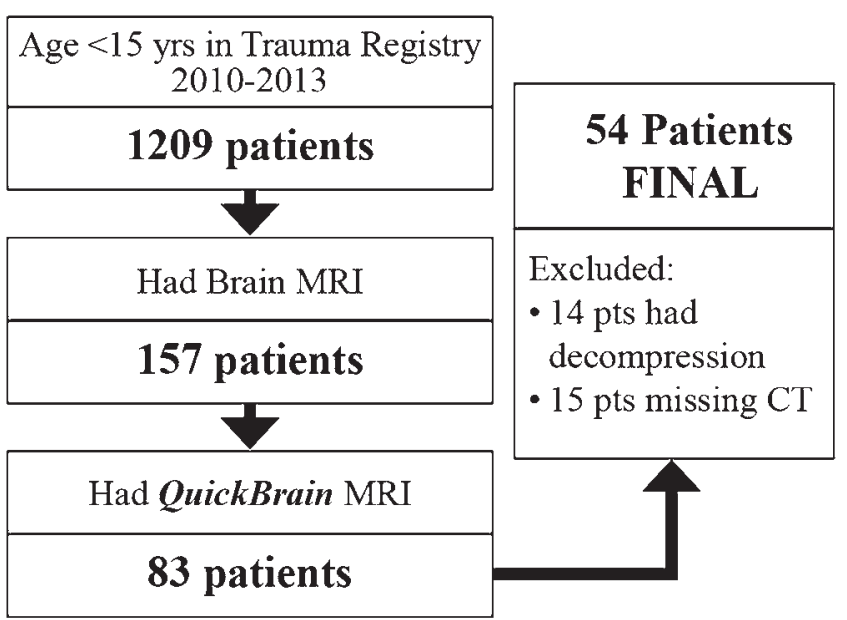

FIG. 1. Patient population. pts $=$ patients.

tal stay. The average time interval between the initial head $\mathrm{CT}$ and qbMRI was 27.5 hours (range, 3.8-146.1 hours), and $41 \%$ occurred within 12 hours (Table 1). No qbMRI was obtained in the ED for initial evaluation.

\section{Intracranial Lesion Identification}

The sensitivity and specificity of qbMRI to detect any radiographic TBI were $85 \%$ (95\% CI 73\%-93\%) and $100 \%$ (95\% CI 61\%-100\%), respectively (Fig. 2; Tables 2 and 3 ). When we performed the analysis using only patients with a ciTBI, the sensitivity increased to $100 \%(95 \%$ CI $89 \%-100 \%)$, and the specificity remained $100 \%(95 \%$ CI $34 \%-100 \%$ ).

\section{Missed Intracranial Lesions}

The unblinded review by the study pediatric neurosurgeon (N.R.S.) of lesions missed on qbMRI by neuroradiology is detailed in Table 4. These reviews occurred after the independent neuroradiology reviews were complete and a lesion was not identified. All except 1 patient were found to have a subgaleal hematoma and skull fracture, which suggests that small epidural hematomas found on initial CT had decompressed into the subgaleal space before follow-up qbMRI. In the 1 additional case, both the head CT and qbMRI were read by the blinded radiologists

TABLE 1. Patient demographics

\begin{tabular}{ll}
\hline \multicolumn{1}{c}{ Demographic } & \multicolumn{1}{c}{ Value } \\
\hline Mean age in yrs (range) & $4.1(0.03-12.3)$ \\
\hline Male sex (\%) & 65 \\
\hline Transferred from outside hospital (\%) & 82 \\
\hline GCS score $\geq 14$ (\% [range]) & $74(3-15)$ \\
\hline ciTBI (\%) & 63 \\
\hline Median hospital LOS in hrs (range) & $66.7(5.5-806.6)$ \\
\hline $\begin{array}{l}\text { Mean delay btwn initial head CT and } \\
\text { qbMRI in hrs (range) }\end{array}$ & $27.5(3.8-146.1)$ \\
\hline
\end{tabular}

* ciTBI was indicated by death, need for a neurosurgical procedure, a hospital LOS of $>2$ days, and/or need for endotracheal intubation for $>1$ day.
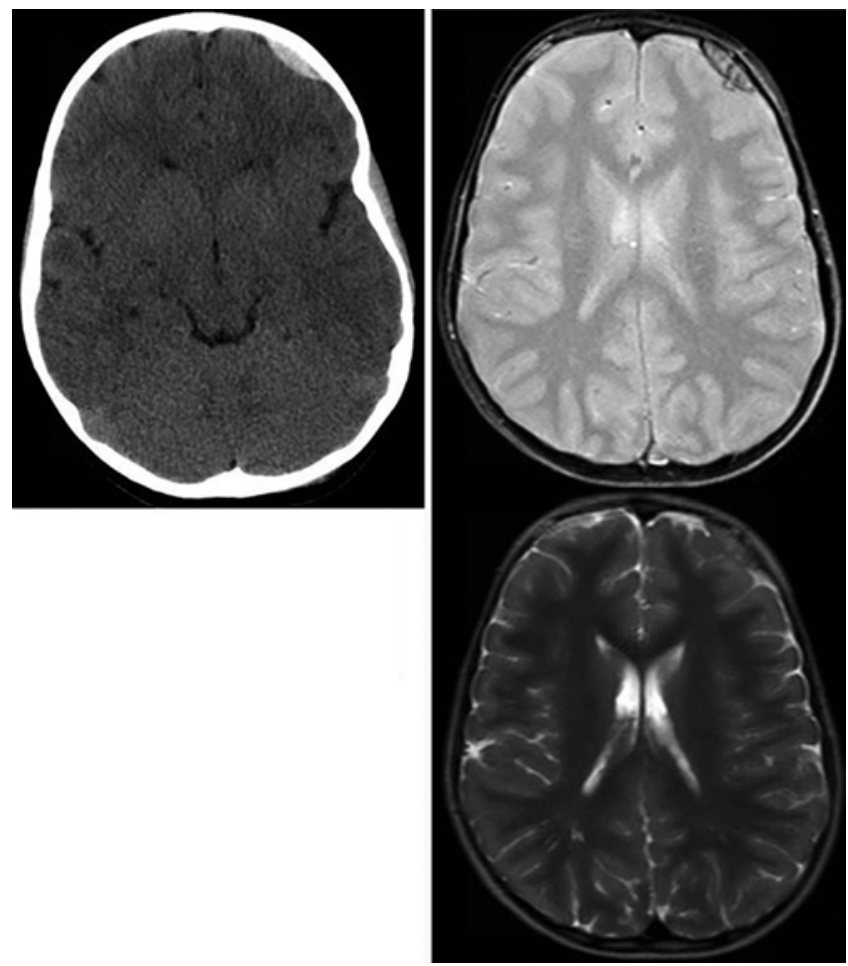

FIG. 2. Comparison images of the same 5-year-old child: head CT scan (left) and qbMR images (right) (two slices) showing epidural hematoma. The interval between head CT and MRI was 6 hours.

as revealing a contusion, but no obvious or clinically significant contusion was seen on either imaging modality retrospectively by the study pediatric neurosurgeon. The average time between qbMRI and CT for missed lesions was 21.6 hours (range 5.1-39.1 hours).

\section{Characterization of Intracranial Bleed Location}

Thirty-eight qbMRI studies were noted to show an intracranial hemorrhage (i.e., excluded contusions, skull fractures, etc.). In these studies, $92 \%$ of the qbMRI studies correctly characterized the lesion as intraaxial instead of extraaxial.

\section{Visualization of Midline Shift}

Midline shift was noted on head CT in 8 patients. The sensitivity and specificity of qbMRI to detect the midline shift were $75 \%$ (95\% CI 41\%-93\%) and 90\% (95\% CI $75 \%-97 \%$ ), respectively (Tables 2 and 5), in patients who had a lesion identified with qbMRI. In the studies identified by radiologists to be qbMRI false negative, the study pediatric neurosurgeon obtained very similar measurements of midline shift in both qbMRI and CT images,

TABLE 2. Sensitivity and specificity of qbMRI

\begin{tabular}{lcc}
\hline \multicolumn{1}{c}{ MRI Result } & Sensitivity (\% [95\% Cl]) & Specificity (\% [95\% CI]) \\
\hline Intracranial lesion & $85(73-93)$ & $100(61-100)$ \\
\hline ciTBI & $100(89-100)$ & $100(34-100)$ \\
\hline Midline shift & $75(41-93)$ & $90(75-97)$ \\
\hline
\end{tabular}


TABLE 3. Injury present on head CT and qbMR images

\begin{tabular}{ccc}
\hline $\begin{array}{c}\text { Injury Present } \\
\text { on qbMRI }\end{array}$ & \multicolumn{2}{c}{ Injury Present on CT (no.) } \\
\cline { 2 - 3 } & Yes & No \\
\hline Yes & 41 & 0 \\
\hline No & 7 & 6 \\
\hline
\end{tabular}

contrary to the radiologist's review. In the studies noted to be qbMRI false positive, the study pediatric neurosurgeon retrospectively noted midline shift in both $\mathrm{CT}$ images and qbMRI in 1 case but agreed that there was no midline shift on the initial CT in 2 cases in which it was seen later in qbMRI. The time intervals between $\mathrm{CT}$ and qbMRI in these latter 2 cases were 15.6 and 22.5 hours, and both patients subsequently underwent a neurosurgical procedure after the qbMRI, which suggests that the qbMRI detected a clinically significant deterioration that altered management (rather than providing more accurate information).

\section{Discussion}

To our knowledge, literature on the accuracy of qbMRI for pediatric head trauma in the acute setting is sparse. Although ours was a small pilot study, the findings support the concept of MRI as a potentially viable alternative to CT for evaluating acute pediatric head trauma. We found qbMRI to be reasonably accurate for detecting pediatric TBI, which suggests that qbMRI has the potential to serve as an alternative to CT when TBI is suspected. However, larger, prospective studies are needed to confirm our findings.

Ionizing radiation from head $\mathrm{CT}$ is an important public health problem. As CT scan availability has become more widespread, there has been a substantial increase in CT use in the United States, and children can be exposed to multiple studies during their lifetime. ${ }^{1,9}$ Studies have estimated a 1 in 1500 to 1 in 5000 patient incidence of cancer from CT scans during a child's life. ${ }^{16}$ In addition, 1 study found that of all CT scans ordered for adolescents, the majority were head CT scans (90\%), and more than 600,000 head and abdominal CT scans were performed annually in children younger than 15 years. ${ }^{3,4}$ The risk of leukemia from $\mathrm{CT}$ of the head is highest in children younger than 5 years, and children in this age group frequently present with head injury, ${ }^{14}$ as supported by the median age of 3 years in our cohort. Attempts to reduce exposure to ionizing radiation in children could include improving education on radiation dosing, reducing the radiation dose delivered by CT scans, and using clinical decision rules designed to reduce imaging rates. ${ }^{8}$ MRI offers an alternative to these strategies and might be more effective in reducing radiation in locations such as the United States, where markedly reducing imaging rates might be challenging.

QuickBrain MRI has been used extensively and is now routine practice at many children's hospitals for the evaluation of ventricular size when a ventriculoperitoneal shunt malfunction is suspected. ${ }^{11,15,21}$ Past studies in adults evaluated MRI as a diagnostic modality for intracranial hemorrhage. 2,6,7,18 MRI studies that include specific sequences, such as susceptibility-weighted imaging (SWI) or gradient echo (GRE), are highly accurate for detecting blood while adding only minutes to the acquisition time; ${ }^{7}$ GRE can detect blood by detecting different states of hemoglobin. ${ }^{2}$ One investigation noted MRI with GRE sequences to be as accurate as head $\mathrm{CT}$ for diagnosing acute intracerebral hemorrhage, and a second study found it to be slightly more accurate than CT in identifying small bleeds. ${ }^{6,18}$ Results of these investigations raise the possibility that the sensitivity and specificity of a rapid MRI protocol could be maximized if these sequences that are specific for blood are added without adding substantially to the imaging time.

A recent study evaluated full-brain MRI within 5 days of acute TBI in a pediatric population and found it to be highly accurate in delineating traumatic lesions. ${ }^{17}$ The authors of that study noted MRI to be as sensitive as CT for identifying intracranial injuries, although skull fractures were missed by MRI in 5 of 13 patients. The authors did not detail the clinical course of each patient with a missed skull fracture, but they did note the rather benign nature of a nondepressed or nondisplaced skull fracture overall. In our study, 2 skull fractures were missed, but they did not require any significant clinical interventions other than admission and observation.

In some hospitals, the feasibility of MRI can be limited because of its physical distance from the ED, limited hours of operation, long delays in access, or the need to

TABLE 4. Diagnosis based on head CT

\begin{tabular}{|c|c|c|c|c|c|c|c|c|}
\hline $\begin{array}{l}\text { Patient } \\
\text { No. }\end{array}$ & Age & Diagnosis & $\begin{array}{l}\text { Lesion } \\
\text { Size }(m m)^{*}\end{array}$ & $\begin{array}{l}\text { Time Delay } \\
\text { (hrs) }\end{array}$ & $\begin{array}{l}\text { Hospital } \\
\text { LOS (hrs) }\end{array}$ & $\begin{array}{l}\text { PICU LOS } \\
\quad(h r s)\end{array}$ & $\begin{array}{l}\text { Procedure } \\
\text { Performed }\end{array}$ & $\begin{array}{l}\text { Results of Neurosurgeon Review of } \\
\text { qbMRI } †\end{array}$ \\
\hline 1 & 3 mos & Skull fracture & NA & 8.6 & 22 & 0 & None & No fracture but subgaleal hematoma \\
\hline 2 & $1.6 \mathrm{yrs}$ & Cerebral contusion & NA & 5.1 & 30 & 0 & None & No abnormalities seen on CT or qbMRI \\
\hline 3 & $5.8 \mathrm{yrs}$ & Epidural hematoma & 5 & 33.9 & 35 & 0 & None & Subgaleal hematoma \\
\hline 4 & $2.5 \mathrm{mos}$ & Subdural hematoma & 4 & 14.2 & 31.8 & 31.8 & None & Subgaleal hematoma \\
\hline 5 & 3 wks & Subdural hematoma & 3 & 11.8 & 41 & 20.1 & None & Subgaleal hematoma \\
\hline 6 & $11 \mathrm{mos}$ & Skull fracture & NA & 39.1 & 39.5 & 18.6 & None & No fracture but subgaleal hematoma \\
\hline 7 & 1 wk & Subdural hematoma & 2 & 38.4 & 46.6 & 22.3 & None & Subgaleal hematoma \\
\hline
\end{tabular}


TABLE 5. Midline shift present on head CT and qbMR images*

\begin{tabular}{ccc}
\hline $\begin{array}{c}\text { Midline Shift Present } \\
\text { on qbMRI (no.) }\end{array}$ & \multicolumn{2}{c}{ Midline Shift Present on CT (no.) } \\
\cline { 2 - 3 } & Yes & No \\
\hline Yes & 6 & 3 \\
\hline No & 2 & 28 \\
\hline
\end{tabular}

* Only patients who had a lesion identified on qbMRI are included here.

sedate the patient to obtain quality images. A recent study evaluated the feasibility of qbMRI for evaluating TBI. ${ }^{5}$ That study evaluated 57 children who underwent qbMRI without any accompanying head CT. The authors found that the scan time for qbMRI was 3-4 minutes and resulted in an average LOS that was comparable with that of children who underwent head CT imaging. Also, previous studies of patients with a ventriculoperitoneal shunt made qbMRI readily accessible in most centers and found that the need for a sedative and anxiolytic (approximately $4 \%$ for both) were similar between children undergoing qbMRI and those undergoing CT. ${ }^{21}$

None of the lesions missed on qbMRI in this study fulfilled the definition of a ciTBI. Of the 7 non-ciTBIs that were missed, an unblinded evaluation of these studies by 1 of the authors revealed that there was a subgaleal hematoma and skull fracture in every case in which the original CT imaging had identified a small epidural hematoma. This finding suggests that the small hematoma seen in the original CT images might no longer have been present on the delayed-interval qbMRI because it had decompressed into the subgaleal space in the time between the scans. Midline shift detection in this trial was $75 \%$, but this number might not reflect the ability of qbMRI to detect this finding. Two of the 3 children for whom the qbMRI did not agree with the CT imaging for midline shift went on to undergo decompressive craniotomy, which suggests that the treating pediatric neurosurgeon felt that compression or shift was present. This result suggests an interval worsening of the intracranial process, that is, not a missed finding attributable to differences in the sensitivity of the imaging modalities but a limitation of the study design because of the intentional time delay between the initial $\mathrm{CT}$ and follow-up qbMRI studies. Future validation of the utility of qbMRI in the evaluation of acute TBI in children should involve contemporaneous $\mathrm{CT}$ and qbMRI so that direct comparison of the status of intracranial lesions can be made.

This study has a number of limitations, including the retrospective study design and relatively modest sample size. The most significant limitation is that the CT and qbMRI studies were obtained intentionally at different time periods for clinical care to evaluate interval changes and progression of bleeding. With this being one of the first studies to evaluate its test characteristics for acute pediatric head trauma, qbMRI was not the standard of care; therefore, this imaging modality was not used as the initial test for any patient in this study. The sensitivity might have been higher if the interval between CT and qbMRI had been shorter. In addition, adding specific sequences, such as GRE, to evaluate for blood might help maximize sensitivity. The final limitation is that this study included patients who were evaluated for TBI and admitted to a hospital. Therefore, the majority of them had an intracranial lesion, which might have introduced selection bias to the study. All patients who did not have an intracranial injury were reviewed as such by the blinded reviewers.

\section{Conclusions}

This pilot study found that qbMRI has reasonable sensitivity for the identification of pediatric TBI in a cohort of children hospitalized for trauma. Additional work in this area and advances in MRI technology might ultimately validate the qbMRI modality for the initial evaluation of children suspected of having TBI, which would further reduce the risk of their exposure to ionizing radiation.

\section{Acknowledgments}

We acknowledge Drs. Joanna Prescott and Joshua Knight for reviewing all images in this study.

\section{References}

1. Berdahl CT, Vermeulen MJ, Larson DB, Schull MJ: Emergency department computed tomography utilization in the United States and Canada. Ann Emerg Med 62:486-494, 494.e1-494.e3, 2013

2. Bradley WG Jr: MR appearance of hemorrhage in the brain. Radiology 189:15-26, 1993

3. Brenner D, Elliston C, Hall E, Berdon W: Estimated risks of radiation-induced fatal cancer from pediatric CT. AJR Am J Roentgenol 176:289-296, 2001

4. Choi SJ, Kim EY, Kim HS, Choi HY, Cho J, Yang HJ, et al: Cumulative effective dose associated with computed tomography examinations in adolescent trauma patients. Pediatr Emerg Care 30:479-482, 2014

5. Cohen AR, Caruso P, Duhaime A-C, Klig JE: Feasibility of "rapid" magnetic resonance imaging in pediatric acute head injury. Am J Emerg Med 33:887-890, 2015

6. Copenhaver BR, Shin J, Warach S, Butman JA, Saver JL, Kidwell CS: Gradient echo MRI: implementation of a training tutorial for intracranial hemorrhage diagnosis. Neurology 72:1576-1581, 2009

7. Di Ieva A, Lam T, Alcaide-Leon P, Bharatha A, Montanera W, Cusimano MD: Magnetic resonance susceptibility weighted imaging in neurosurgery: current applications and future perspectives. J Neurosurg 123:1463-1475, 2015

8. Donnelly LF, Emery KH, Brody AS, Laor T, Gylys-Morin VM, Anton CG, et al: Minimizing radiation dose for pediatric body applications of single-detector helical CT: strategies at a large Children's Hospital. AJR Am J Roentgenol 176:303-306, 2001

9. Hahn B, Dima J, Hirschorn D, Weiserbs KF: Incidence and pathology of repeat computed tomography of the abdomen and pelvis in a pediatric emergency department population. Pediatr Emerg Care 29:822-825, 2013

10. Holmes JF, Borgialli DA, Nadel FM, Quayle KS, Schambam N, Cooper A, et al: Do children with blunt head trauma and normal cranial computed tomography scan results require hospitalization for neurologic observation? Ann Emerg Med 58:315-322, 2011

11. Iskandar BJ, Sansone JM, Medow J, Rowley HA: The use of quick-brain magnetic resonance imaging in the evaluation of shunt-treated hydrocephalus. J Neurosurg 101 (2 Suppl):147-151, 2004

12. Kuppermann N, Holmes JF, Dayan PS, Hoyle JD Jr, Atabaki SM, Holubkov R, et al: Identification of children at very low 
risk of clinically-important brain injuries after head trauma: a prospective cohort study. Lancet 374:1160-1170, 2009

13. Lee B, Newberg A: Neuroimaging in traumatic brain imaging. NeuroRx 2:372-383, 2005

14. Miglioretti DL, Johnson E, Williams A, Greenlee RT, Weinmann S, Solberg LI, et al: The use of computed tomography in pediatrics and the associated radiation exposure and estimated cancer risk. JAMA Pediatr 167:700-707, 2013

15. Missios S, Quebada PB, Forero JA, Durham SR, Pekala JS, Eskey CJ, et al: Quick-brain magnetic resonance imaging for nonhydrocephalus indications. J Neurosurg Pediatr 2:438444, 2008

16. Pearce MS, Salotti JA, Little MP, McHugh K, Lee C, Kim $\mathrm{KP}$, et al: Radiation exposure from CT scans in childhood and subsequent risk of leukaemia and brain tumours: a retrospective cohort study. Lancet 380:499-505, 2012

17. Roguski M, Morel B, Sweeney M, Talan J, Rideout L, Riesenburger RI, et al: Magnetic resonance imaging as an alternative to computed tomography in select patients with traumatic brain injury: a retrospective comparison. J Neurosurg Pediatr 15:529-534, 2015

18. Romanova AL, Nemeth AJ, Berman MD, Guth JC, Liotta EM, Naidech AM, et al: Magnetic resonance imaging versus computed tomography for identification and quantification of intraventricular hemorrhage. J Stroke Cerebrovasc Dis 23:2036-2040, 2014

19. Smitherman E, Hernandez A, Stavinoha PL, Huang R, Kernie SG, Diaz-Arrastia R, et al: Predicting outcome after pediatric traumatic brain injury by early magnetic resonance imaging lesion location and volume. J Neurotrauma 33:35-48, 2016
20. Thompson EM, Baird LC, Selden NR: Results of a North American survey of rapid-sequence MRI utilization to evaluate cerebral ventricles in children. J Neurosurg Pediatr 13:636-640, 2014

21. Yue EL, Meckler GD, Fleischman RJ, Selden NR, Bardo DM, Chu O'Connor AK, et al: Test characteristics of quick brain MRI for shunt evaluation in children: an alternative modality to avoid radiation. J Neurosurg Pediatr 15:420426, 2015

\section{Disclosures}

The authors report no conflict of interest concerning the materials or methods used in this study or the findings specified in this paper.

\section{Author Contributions}

Conception and design: Sheridan, Newgard, Selden, Hansen. Acquisition of data: Sheridan. Analysis and interpretation of data: Sheridan, Hansen. Drafting the article: Sheridan. Critically revising the article: all authors. Reviewed submitted version of manuscript: all authors. Approved the final version of the manuscript on behalf of all authors: Sheridan. Statistical analysis: Sheridan. Administrative/technical/material support: Sheridan. Study supervision: Sheridan, Hansen.

\section{Correspondence}

David C. Sheridan, Department of Emergency Medicine, Oregon Health \& Science University, 707 SW Gaines Rd., Mail Code CDRC-W, Portland, OR 97239. email: sheridda@ohsu.edu. 